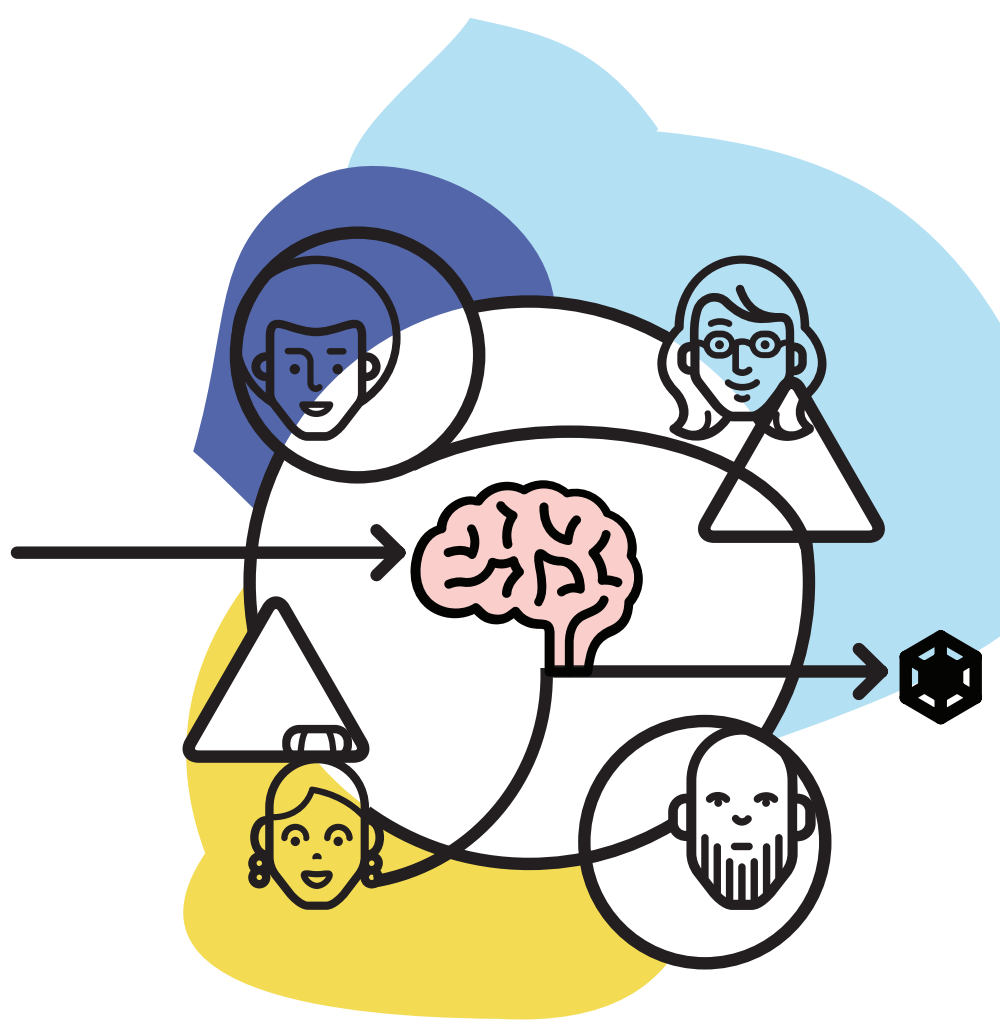

\title{
DISEÑO Y DESARROLLO \\ DE UN SISTEMA DE GESTIÓN DEL CONOCIMIENTO EN EL LATU
}

\author{
AUTORES \\ STELLA CRISTÓBAL (1) \\ CARINA DI CANDIA (1) \\ GABRIELA MADERNI (1)
}

(1) GERENCIA DE TECNOLOGÍA Y GESTIÓN, LATU ste artículo describe el proyecto de diseño e implementación del sistema de gestión del conocimiento $(\mathrm{GdC})$ en el Laboratorio Tecnológico del Uruguay (LATU), entre los años 2014 y 2016.

La implementación del sistema de GdC es parte de los objetivos estratégicos del LATU, en tanto organización de derecho público no estatal cuya misión es impulsar el desarrollo sustentable del país y su inserción internacional a través de la innovación y la transferencia de soluciones de valor. 
El proceso permitió visualizar cómo un sistema de gestión del conocimiento diseñado a medida, con participación del personal, compartiendo con ellos un lenguaje técnico común, respetando su cultura y sus tiempos y habilitando el intercambio entre los integrantes en busca de consensos en la toma de las decisiones críticas, permite la construcción de un sistema adecuado a las necesidades, fortalezas y debilidades de la organización que lo implementa.

A partir de los resultados del proyecto de implementación se infieren conclusiones que pueden ser de utilidad para toda organización que quiera o se encuentre transitando por un proyecto de este tipo.

\section{En contexto}

En la llamada "economía del conocimiento" los centros tecnológicos y de investigación tienen un rol protagónico (Organisation for Economic Co-operation and Development, OECD, 2000; Organisation for Economic Co-operation and Development, OECD, 2004). Son productores de conocimiento, un activo económico capaz de promover un crecimiento sostenible y una ventaja competitiva decisiva para las empresas y los países. El conocimiento y la capacidad de aprender se han convertido en los factores fundamentales de creación de valor de una organización.

Tradicionalmente, las organizaciones han invertido recursos y esfuerzos en optimizar su gestión en diversos campos, entre ellos el administrativo, el comercial, los recursos humanos, la calidad y los riesgos. En los últimos años ha tomado relevancia la eficaz y eficiente gestión del conocimiento. Para ello es necesario desarrollar una cultura de colaboración y de conocimiento institucional compartido, puesto que la gestión del conocimiento es el esfuerzo de capturar y sacar provecho de la experiencia colectiva de la organización haciéndola accesible a sus integrantes.

Como disciplina empresarial, la gestión del conocimiento es relativamente reciente. Sus pilares académicos más destacables, los primeros modelos que brindaron un marco teórico completo, se remontan a la última década del siglo pasado (Wiig, 1993; Nonaka y Takeuchi, 1995; Boisot, 1998).

EI LATU es consciente de la relevancia que el conocimiento organizacional ha tenido a lo largo de su vida institucional y de lo vital que será en el futuro optimizar su gestión para cumplir su misión en la actual economía del conocimiento. Hasta el momento, no existe en nuestro país ningún antecedente documentado a nivel académico de una implementación de esta naturaleza. Incluso a nivel internacional, los ejemplos son muy escasos. Esto motiva la difusión de la experiencia y de una posible metodología de intervención replicable en otras organizaciones uruguayas.

\section{La gestión del conocimiento}

Según la bibliografía, existen más de 100 definiciones publicadas de la gestión del conocimiento $(\mathrm{GdC})$, que pueden ser distinguidas según la perspectiva con la que se identifican: la perspectiva empresarial, la perspectiva cognitiva y la perspectiva de proceso o tecnológica.

La perspectiva empresarial considera el conocimiento como un componente de las actividades empresariales con un interés explícito reflejado en la estrategia, en la política y en la práctica a todo nivel de la organización, y establece una conexión directa entre el capital intelectual de la organización y los resultados positivos del negocio. En esta perspectiva, la GdC es un acercamiento colaborador e integrador en relación a la creación, captura, organización, acceso y uso del capital intelectual de la empresa.

En palabras de Lee Blanco (2012): “A diferencia de la economía tradicional, sostenida en bienes tangibles, la nueva Economía se basa en bienes intangibles. En ese marco el concepto de capital intelectual adquiere relevancia y es entendido como una nueva forma de medición del valor de una organización, con fines de lucro o no, que influye no solamente en la economía sino en la sociedad en general".

Para la perspectiva cognitiva el conocimiento es la fuente fundamental que nos permite funcionar inteligentemente. Con el tiempo, una gran cantidad de conocimiento se ha transformado en otras manifestaciones, como libros, tradiciones, tecnología, etcétera. Estas transformaciones resultan en un cúmulo de experiencias y cuando son utilizadas apropiadamente incrementan la efectividad. El conocimiento es el principal factor que posibilita a nivel personal, organizacional y de la sociedad que el comportamiento inteligente sea posible (Wiig, 1993).

Para la perspectiva de proceso o tecnológica, la GdC supone una gestión sistemática del uso de la información, con el objetivo de proveer una base continua de conocimiento a las personas correctas en el momento preciso, para que puedan tomar decisiones efectivas y eficientes en el día a día empresarial. 
Es decir, en un extremo, la gestión del conocimiento comprende todo lo que tiene que ver con conocimiento. En el otro, es básicamente definida como un sistema tecnológico de información que brinda el know how organizacional. Sin embargo, hay consensos entre los diversos autores consultados sobre el campo altamente multidisciplinario de la GdC.

Una popular y errónea interpretación de la GdC la focaliza en transformar el conocimiento tácito en una forma más explícita o tangible, para luego almacenarlo o archivarlo de alguna forma, usualmente en una intranet o un portal de conocimiento. Esta interpretación es asumida por todas aquellas organizaciones que generan un vasto inventario de conocimiento tangible y lo hacen accesible a todos los empleados sin haber realizado un análisis de necesidades para identificar cómo el compartir conocimiento puede beneficiar a los individuos, los grupos y a la organización en su globalidad. Asumen que los empleados utilizarán masivamente el conocimiento que ponen a su disposición, lo cual generalmente no sucede.

La GdC contribuye a aumentar la capacidad competitiva de una organización, a lograr una actividad de innovación sostenida y a aumentar su eficiencia, poniendo más énfasis en el factor humano que en el material. Pero debe diseñarse sobre una filosofía de captura del conocimiento y de promoción de una cultura organizacional que pase de la noción del atesoramiento del conocimiento, como factor de poder, a compartir el conocimiento. Según esta concepción, el atesorar conocimiento no es poder; el poder está en compartirlo, en ser capaces de generar valor a partir de un conocimiento que se genera con el agregado de distintas inteligencias (Dalkir, 2005).

En la actualidad, se puede decir que la importancia de implementar una adecuada gestión del conocimiento en una organización radica en cuatro evidencias clave:

- La globalización de los negocios.

- La necesidad de contar con "organizaciones magras" en las que los trabajadores del conocimiento trabajen más inteligentemente incorporando conocimientos en forma permanente, mejorando la efectividad de la organización.

- Lo que suele denominarse "amnesia corporativa", debido a una mayor movilidad laboral y falta de comunicación entre las distintas áreas o procesos, que genera problemas de continuidad del conocimiento dentro de una organización y permanente demanda de capacitación.

- Los avances tecnológicos, principalmente rela- cionados con las tecnologías de la información, que han cambiado radicalmente las expectativas en relación a los tiempos de respuesta y generan entornos laborales con sobrecarga de información.

La GdC no solo debe gestionar la información que se ha explicitado sino también aquella que permanece como conocimiento tácito. No siempre las organizaciones son conscientes de lo que no tienen conocimiento.

Newell (2005) identifica las tres principales características del conocimiento que dificultan su creación y transferencia y, por lo tanto, su gestión: la dispersión, la ambigüedad y la perturbación del conocimiento. La dispersión del conocimiento está relacionada a que distintas personas/grupos son expertos en diferentes ámbitos y con frecuencia no saben qué es lo que otros saben; la ambigüedad hace referencia a que distintas personas/grupos atribuyen diferentes significados a los mismos conceptos, y la perturbación, a que distintas personas/grupos temen perder su poder si comparten lo que saben. Es por ello que la transferencia de conocimiento puede estar suboptimizada como consecuencia de la posible pérdida de poder y estatus.

Este tipo de perturbación ocasionada por la naturaleza compleja del conocimiento suele denominarse perturbación inducida por el poder. En las organizaciones en que la $\mathrm{GdC}$ requiere cambios significativos de la conducta y de la cultura imperantes es posible detectar lo que Newell (2005) denomina perturbación inducida por la cultura. Una tercera forma de perturbación está relacionada con la falta de tiempo del personal para desarrollar tareas relacionadas con la GdC, adicionales al trabajo normal. A esta perturbación el autor la denomina perturbación inducida por las tareas. Los tres tipos de perturbación se interrelacionan dinámicamente.

Snowden (2002) considera que la sociedad se encuentra transitando la tercera ola de la gestión del conocimiento, luego de atravesar dos períodos anteriores muy bien caracterizados de acuerdo a los énfasis de la época:

- En la primera generación de la GdC el énfasis estaba dado en los contenedores de conocimiento o de tecnologías de la información. Durante esta primera generación fueron implementados un gran número de intranets y sistemas internos de gestión del conocimiento. Se buscó detectar la mayor cantidad de información que estuviera "enterrada" en la organización y recuperarla a través de "medios encapsulados recuperables". 
- La segunda generación de la GdC se focalizó en las personas. Existía un gran reconocimiento sobre la importancia de la dimensión humana y cultural de la GdC. Surgen entonces las comunidades de práctica, un buen vehículo para compartir conocimiento o estudiar el movimiento del conocimiento a través de la organización, lo que permite mejorar la eficiencia de la organización, y crear conocimiento para incentivar la innovación.

- La tercera generación de la GdC, la actual, es consciente de la importancia de compartir el contexto: cómo describir y organizar los contenidos para que sus usuarios sepan que existen y puedan fácilmente acceder a ellos y aplicarlos. Esta tercera generación se caracteriza por el uso del metadata para describir el contenido. La gestión del conocimiento debe satisfacer las necesidades individuales, de las comunidades de práctica y de la organización, de lo contrario, no será exitosa.

En esta tercera ola los beneficios de realizar una efectiva $\mathrm{GdC}$ se comprueban a nivel individual, de las comunidades de práctica y de toda la organización. La Tabla 1 resume algunos de estos beneficios (Dalkir, 2005).

Entre los desafíos críticos de la GdC se identifican los relacionados a gestionar los contenidos en forma efectiva, facilitar la colaboración, contribuir al relacionamiento de los trabajadores del conocimiento, identificando los expertos y colaborando con la retención de talentos, y, finalmente, ayudar a la organización a tomar decisiones basadas en datos, información y conoci- mientos completos, válidos y bien interpretados (Asociación Española de Normalización y Certificación, 2008).

\section{El ciclo integrador de la gestión del conocimiento}

El denominado ciclo integrador de la gestión del conocimiento (Figura 1) resulta de la comparación que realiza Kimiz Dalkir en su libro Knowledge Management in Theory and Practice (2005), de los ciclos de gestión del conocimiento de varios autores (Meyer y Zack, 1996; Bukowitz y Williams, 2000; Mc Elroy, 2003; Wiig, 1993).

En el ciclo integrador de la gestión del conocimiento se diferencian tres grandes etapas:

- Capturar y/o crear conocimiento.

- Compartir y diseminar el conocimiento.

- Comprender y aplicar el conocimiento.

En la transición de la etapa de conocimiento capturado/ creado a la etapa de conocimiento compartido y diseminado, el conocimiento es evaluado y tasado. Luego, el conocimiento es contextualizado para poder ser comprendido y utilizado. Después de esta tercera etapa se reinicia la primera, y se enriquece así la base de conocimiento inicial. La captura de conocimiento corresponde a la identificación y posterior codificación del conocimiento interno o externo a la organización (desconocido hasta ahora por ella) y de su know how. La creación de

\begin{tabular}{|c|c|c|}
\hline Individual & De las comunidades de práctica & De la organización \\
\hline $\begin{array}{l}\text { - Ayuda a las personas a estar } \\
\text { actualizadas y realizar sus trabajos } \\
\text { en forma más productiva y creati- } \\
\text { va, tomando mejores decisionesy } \\
\text { resolviendo problemas. } \\
\text { - Construye lazos de relaciona- } \\
\text { miento dentro de la organización. } \\
\text { - Contribuye a los desafíos y } \\
\text { genera oportunidades. }\end{array}$ & $\begin{array}{l}\text { - Desarrolla habilidades profesionales. } \\
\text { - Promueve el relacionamiento de } \\
\text { igual-igual (peer-to-peer mentoring). } \\
\text { - Facilita redes de trabajo más efec- } \\
\text { tivas y colaborativas. } \\
\text { - Desarrolla códigos de ética profe- } \\
\text { sional que los miembros de la co- } \\
\text { munidad deben seguir. } \\
\text { - Desarrolla un lenguaje común. }\end{array}$ & $\begin{array}{l}\text { - Ayuda a la estrategia. } \\
\text { - Resuelve problemas rápidamente. } \\
\text { - Difunde las mejores prácticas. } \\
\text { - Mejora la aplicación de conocimiento a } \\
\text { los productos y servicios. } \\
\text { - Ayuda al intercambio de ideas, generan- } \\
\text { do un ambiente propicio para la innovación. } \\
\text { - Permite a la organización estar en } \\
\text { una mejor posición en cuanto a sus } \\
\text { competidores. } \\
\text { - Construye la memoria organizacional. }\end{array}$ \\
\hline
\end{tabular}

Tabla 1. Beneficios de la gestión del conocimiento. Fuente: elaboración propia con base en bibliografía consultada (Dalkir, 2005; Snowden, 2002). 


\section{CULTURA ORGANIZACIONAL}
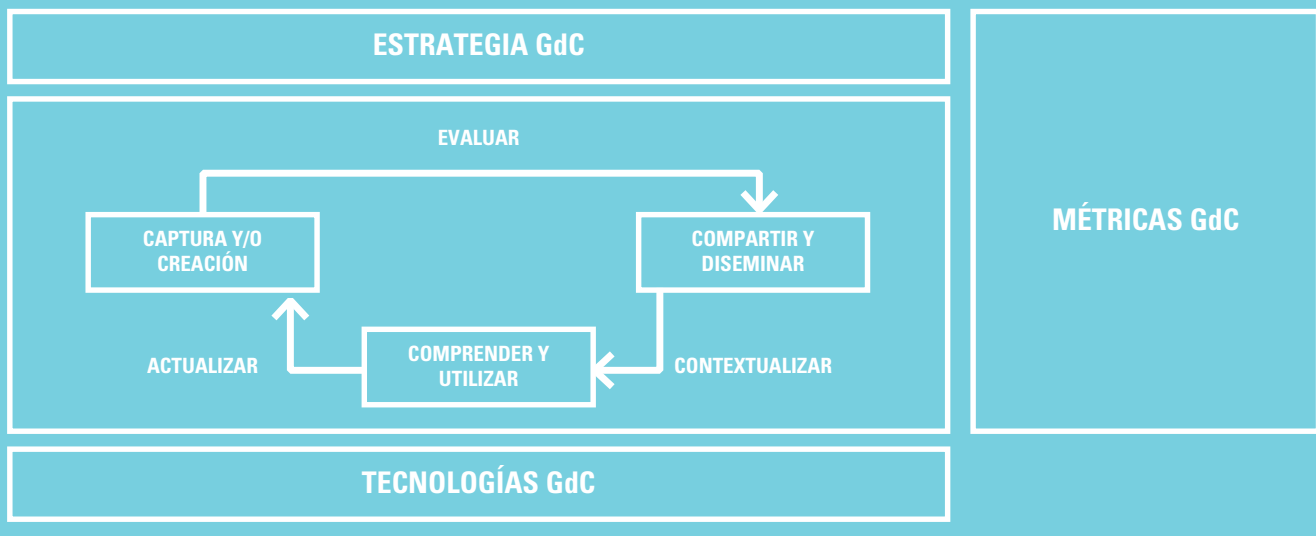

\section{TECNOLOGÍAS GdC}

Figura 1. Ciclo integrador de la gestión del conocimiento. Fuente: traducido de Kimiz Dalkir, 2005, Figura 10-1, página 284.

conocimiento es el desarrollo de nuevo conocimiento, know how e innovaciones que no tenían existencia previa en la organización.

Cuando el conocimiento ya ha sido inventariado en esta primera etapa, es crítico establecer el criterio de selección y su clasificación (tasación), los cuales deben estar alineados con los objetivos organizacionales para determinar si el conocimiento inventariado aporta valor a la organización como para ser incorporado a su capital intelectual.

Una vez que se ha determinado que el nuevo contenido es de valor, la fase siguiente es contextualizarlo. Esto implica mantener el enlace entre el conocimiento incorporado y las personas que deben necesariamente tener acceso a él: el autor o creador de la idea, los expertos sobre la temática y también aquellos que han acumulado experiencia significativa en el uso de ese contenido. La contextualización implica, además, identificar los atributos clave del contenido para facilitar el acceso a él.

Para la gestión del conocimiento existe actualmente una variedad de herramientas y plataformas. Algunas permiten capturar y representar contenidos para que el acceso sea eficiente, otras permiten descubrir y extraer conocimiento, otras facilitan la generación de la red/malla social y la creación de comunidades y algunas brindan soportes de la comunicación y la colaboración entre grupos.
Ruggles (1997) propone una clasificación de las tecnologías de gestión del conocimiento como herramientas que:

- Contribuyen y mejoran la generación, codificación y transferencia de conocimiento;

- "generan" conocimiento (por ejemplo, Data Mining, que descubre nuevos patrones en datos); codifican el conocimiento de forma de hacerlo accesible a otros, y

- transfieren conocimiento disminuyendo los problemas de tiempo y espacio en la comunicación organizacional.

El ciclo integrador de gestión del conocimiento tiene, además, una dimensión relacionada con la estrategia de gestión del conocimiento (estrategia $\mathrm{GdC}$ ), que debe estar ligada con los objetivos organizacionales, y una segunda dimensión consistente en el marco de paramétricas bajo el cual se monitorea el progreso de los objetivos organizacionales en relación a la gestión del conocimiento (métricas GdC).

Generalmente, los dos objetivos usuales de la gestión del conocimiento son la innovación y la reutilización del conocimiento. La innovación está relacionada en mayor medida a la generación de nuevo conocimiento y de nuevas relaciones entre el conocimiento ya existente. La innovación es el resultado de un gran cúmulo de experiencias, positivas y negativas de lo que ha podido funcionar o no en el pasado (Dalkir, 2005): no ocurre en forma aislada. Una perspectiva errónea de la reutilización es relacionarla con actividades rutinarias, aburridas 
y a trabajo no productivo, ya que el reúso es la base del aprendizaje organizacional. Es más adecuado identificar la reutilización como la diseminación de la innovación.

El nuevo conocimiento bajo la forma de innovación eventualmente es incorporado a la memoria organizacional y se transforma en objeto de ser reutilizado, para que el beneficio de este nuevo conocimiento, el know how, sea diseminado dentro de la organización.

La estrategia de la gestión del conocimiento debe proveer la estructura que asegure esa ganancia de aprendizaje organizacional y su mejora continua, para lograr que no se pierda tiempo repitiendo errores y que todos los integrantes de la organización sean conscientes de las nuevas y mejores formas de pensar y hacer. En este proceso puede surgir un gran número de conocimientos fundamentales, como "productos secundarios", que deben ser reconocidos como tales e inventariados como conocimientos. Pueden estar relacionados a elementos tangibles, como las patentes, o a elementos intangibles, como las competencias esenciales.

La estrategia de gestión del conocimiento debería incluir el marco en el cual se va a realizar el monitoreo de su progreso en pos de alcanzar los objetivos definidos, a lo que se suele denominar las métricas de la gestión del conocimiento. Tres de las métricas más típicas son: el benchmarking (comparación con los mejores), el Cuadro de Mando Integral (dimensión financiera, dimensión clientes, dimensión aprendizaje, crecimiento e innovación y dimensión procesos internos del negocio) y la denominada casa de la calidad (Mazur, 1993).

\section{La cultura organizacional y la gestión del conocimiento}

La bibliografía consultada en la temática busca caracterizar la cultura imperante en una organización teniendo en cuenta que da las pautas de cómo una persona debe comportarse dentro de la organización.

Es evidente que la cultura organizacional es un factor que juega un rol fundamental en la gestión del conocimiento: "Las organizaciones modernas, enfocadas a permanecer y ser cada día más competitivas, deben estar atentas a las necesidades del entorno, reaccionar en forma rápida y dinámica, diseñando e implementando transformaciones internas que permitan eliminar o disminuir el efecto de las amenazas y aprovechar las oportunidades del entorno" (Di Candia, et al., 2011), por ello la importancia de conocer la cultura organizacional, que permitirá una buena implementación de gestión del conocimiento.

Esta gestión requiere de un cambio cultural que promueva el compartir el conocimiento y la colaboración entre los integrantes de la organización (Gruber, 2000), independientemente del tipo de cultura imperante.

Una cultura de compartir conocimiento es aquella en la cual hacerlo es la norma y no la excepción, donde las personas son motivadas a trabajar en forma conjunta, a colaborar, compartir y son premiadas por ello. El paradigma de "conocimiento es poder" debe ser superado y sustituido por el de "compartir conocimiento es aún más poderoso".

La cultura organizacional suele ser más potente que cualquier tecnología comunicacional que pueda ser implementada para promover el compartir conocimiento. Tradicionalmente, el conocimiento tiene un flujo vertical que coincide con el organigrama organizacional.

Sin embargo, hoy en día las organizaciones deben cambiar su cultura organizacional hacia una que también promueva el flujo horizontal de conocimiento. Los sistemas de comunicación pueden ser visualizados como elementos de diseminación de la cultura organizacional (Bloom, 2000).

Sveiby y Simons (2002) sugieren que un clima colaborativo es uno de los principales factores que influencian la efectividad de los trabajadores del conocimiento; concluyeron que el grado en que la cultura organizacional es colaborativa puede ser medido y que el resultado de esa medición es un excelente indicador de cuán exitosa puede llegar a ser la gestión del conocimiento en una organización. Su estudio identificó que la distancia es un factor negativo para la colaboración, es decir, a mayor dispersión de una organización, menor es el clima organizacional de colaboración. Además, el medio de trasmisión del conocimiento tácito más popular es el mano a mano (90\% de los encuestados) seguido de las redes informales (25\%). Algunos de los factores que dificultan el compartir conocimiento tácito incluye la noción de que el conocimiento es poder, el no conocer quién es el experto que posee el conocimiento, el no saber si el conocimiento existe y la pérdida de conocimiento cuando las personas dejan la organización. 
Para la GdC la cultura organizacional ideal es aquella en la cual se enfatiza la comunicación y la coordinación entre los grupos, estimulando así la interacción complementaria del conocimiento tácito y explícito. Una cultura donde los expertos comparten su conocimiento y se promueve el compartir conocimiento a todo nivel jerárquico de la organización, a través de mecanismos de reconocimiento y de recompensa, y ese hábito se incorpora como prioridad en los objetivos corporativos e individuales.

Gruber (2000) ha concluido que para que un medio ambiente organizacional sea un buen soporte del compartir conocimiento debe presentar las siguientes características:

- Una estructura de premios, como reconocimiento al compartir conocimiento con los pares;

- una cultura de apertura y transparencia, donde las agendas sean abiertas;

- una estructura para compartir, fomentando la comunicación y coordinación con los grupos;

- confianza, para que se compartan objetivos, y

- apoyo de la alta dirección, que fomenta comunicaciones ascendentes y descendentes.

\section{Los problemas del aprendizaje organizacional}

Las organizaciones tienen capacidad de aprender gracias a los individuos que las componen. Por lo tanto, los problemas del aprendizaje organizacional estarán muy correlacionados con los problemas de los propios individuos de aprender y de tener una visión global de su aportación y participación en todo el entramado organizativo.

Para Senge (1990), son básicamente siete los problemas relacionados con el aprendizaje dentro de una organización:

- Yo soy mi puesto: lealtad a la tarea, confusión con la identidad y total especialidad. Olvido del propósito de la empresa. Delimitación de zonas de influencia. No hay responsabilidad compartida, por lo que se generan lagunas de actuación cuando interactúan diferentes partes de la organización.

- El enemigo externo: culpar siempre a otros de los problemas de la organización.

- La ilusión de hacerse cargo: la necesidad de hacerse cargo para enfrentar problemas complejos.
Confundir la proactividad con reactividad disfrazada. Controlar lo incontrolable. Controlar desde la distancia.

- La fijación en los hechos: Creer que para cada hecho hay una causa obvia e inmediata. Énfasis en los acontecimientos inmediatos, en producir respuestas inmediatas.

- La parábola de la rana hervida: su aparato interno para detectar amenazas a la supervivencia está preparado para cambios repentinos en el medio ambiente, no para cambios lentos y graduales.

- La ilusión de que "se aprende con la experiencia". Cuando nuestros actos tienen consecuencias que trascienden el horizonte de aprendizaje, se vuelve imposible aprender de la experiencia directa ya que nunca experimentamos directamente las consecuencias de muchas de nuestras decisiones.

- El mito del equipo administrativo: "La mayoría de los equipos administrativos ceden bajo presión. El equipo puede funcionar muy bien con los problemas rutinarios. Pero cuando enfrenta problemas complejos que pueden ser embarazosos o amenazadores, el espíritu de equipo deja de existir" (Chris Argyris citado por Senge, 1990, pp. 37).

\section{Modelos de madurez para la gestión del conocimiento}

Un modelo de madurez es un modelo de evaluación de los procesos de una organización, relacionado con su capacidad para producir los resultados deseados de forma predecible, controlable y confiable. Son modelos que facilitan la evaluación de los procesos y que permiten la comparación de los resultados obtenidos de acuerdo a una escala de valoración.

El primer modelo de madurez denominado Capability Maturity Model (CMM) fue creado para los procesos de desarrollo de software (Harmon, 2009). Fue desarrollado inicialmente por la Universidad Carnegie-Mellon para el Sofware Engineering Institute en 1995. Describe cinco niveles (inicial, repetible, definido, gerenciado y optimizado) y sus características, según se detalla en la Tabla 2. 


\begin{tabular}{|l|c|l|}
\hline \multicolumn{2}{|c|}{ Nivel de madurez } & \\
\hline 1 & Inicial & Los procesos son ad hoc, caóticos y están raramente definidos. \\
\hline 2 & Repetible & Están establecidos los procesos básicos y se siguen los procedimientos definidos. \\
\hline 3 & Definido & $\begin{array}{l}\text { Todos los procesos están definidos, documentados, estandarizados e integrados, } \\
\text { conformando una red. }\end{array}$ \\
\hline 4 & Gerenciado & $\begin{array}{l}\text { Los procesos se miden mediante la recopilación de datos de productividad (eficacia } \\
\text { y eficiencia) y calidad. }\end{array}$ \\
\hline 5 & Optimizado & $\begin{array}{l}\text { Mejora continua de los procesos. Uso intensivo de las métricas. } \\
\text { Se gestiona el proceso de innovación. }\end{array}$ \\
\hline
\end{tabular}

Tabla 2. Niveles de madurez del CMM. Traducción propia de Dalkir (2005).

Gran parte de los modelos de madurez de GdC tienen una estructura básica similar al modelo CMM integrado por tres componentes: niveles, descripción o características de esos niveles y áreas clave.

La mayoría de los modelos de madurez para la GdC actualmente en práctica identifican áreas de procesos clave relacionadas con personas, procesos y tecnologías (Dalkir, 2005).

El área de personas incluye aspectos relacionados con la cultura, las estrategias y las políticas asociadas con los recursos humanos de la organización.

El área de procesos refiere a los aspectos relacionados con los procesos de GdC: creación, recopilación, organización, transferencia y utilización del conocimiento.

El área de tecnología se relaciona con la infraestructura tecnológica de soporte a los procesos, aplicaciones o servicios de GdC y la actitud hacia las TI (Tecnologías de la Información) asociadas.

Pee et al. (2006) proponen el Modelo General de Madurez de GdC (General Knowledge Management Maturity Modell. Es un modelo descriptivo en el cual se definen los atributos esenciales que caracterizan a una organización en un nivel particular de la madurez de la GdC. También es un modelo normativo en el que las prácticas principales caracterizan los tipos ideales de ambientes que deberían esperarse en una organización que se encuentre implementando GdC. La traducción de su contenido se detalla en la Tabla 3.

El instrumento de evaluación proporcionado por este modelo puede ser utilizado como una herramienta de diagnóstico, identificando los aspectos que requieren ser mejorados. Esto permite determinar las actividades esenciales y sus prioridades e indica cómo avanzar al siguiente nivel de madurez de GdC.
Además, puede servir de base para la comparación de las unidades dentro de una organización o entre organizaciones.

\section{Diseño e implementación del Sistema de Gestión del Conocimiento en el LATU}

El Laboratorio Tecnológico del Uruguay (LATU) es una institución de alto prestigio en nuestro país. Creada en la década de 1960 con el fin de realizar el control de calidad de los productos industriales de exportación, por aproximadamente 30 años tuvo escasa visibilidad pública. Sus clientes eran básicamente la industria exportadora de alimentos procesados y de productos derivados de la industria del cuero, y los importadores de alimentos. A partir de la década de 1990, esa situación se fue modificando gradualmente por la incorporación de nuevos servicios relacionados con la certificación de sistemas de gestión de la calidad, las tecnologías de la información, el intercambio tecnológico, industrial y comercial, la muestra interactiva de ciencia y tecnología, la creación de una incubadora de emprendedores con proyectos de base tecnológica y de un centro de desarrollo del conocimiento, y la realización de proyectos con alto impacto en la sociedad, como el Plan Ceibal (del cual el LATU tuvo la implementación técnica y operativa entre los años 2006 y 2010) (Uruguay, 2010).

En función de los cambios que han ocurrido y continuarán ocurriendo en el LATU, ligados básicamente al crecimiento de la institución y al establecimiento de nuevas políticas en relación a los recursos humanos (retiro de técnicos con muchos años de experiencia por causales jubilatorias o personales, política institucional 


\begin{tabular}{|c|c|c|c|c|c|}
\hline \multirow{2}{*}{\multicolumn{2}{|c|}{ Nivel de madurez }} & \multirow{3}{*}{\begin{tabular}{l}
\multicolumn{1}{c}{ Descripción general } \\
Poca o ninguna \\
intención de usar el \\
conocimiento.
\end{tabular}} & \multicolumn{3}{|c|}{ Áreas clave } \\
\hline & & & \multirow{2}{*}{$\begin{array}{l}\text { Personas/organización } \\
\text { La organización no es } \\
\text { consciente de la necesi- } \\
\text { dad de la GdC. }\end{array}$} & \multirow{2}{*}{\begin{tabular}{l}
\multicolumn{1}{c}{ Procesos } \\
No existen proce- \\
sos formales para \\
capturar, compartir \\
y reutilizar el cono- \\
cimiento.
\end{tabular}} & \multirow{2}{*}{\begin{tabular}{l}
\multicolumn{1}{c}{ Tecnología } \\
Sin tecnologías o \\
infraestructura de \\
soporte u apoyo.
\end{tabular}} \\
\hline 1 & Inicial & & & & \\
\hline 2 & Conciencia & $\begin{array}{l}\text { La organización es } \\
\text { consciente y tiene la } \\
\text { intención de la GdC, } \\
\text { posiblemente no sabe } \\
\text { el cómo. }\end{array}$ & $\begin{array}{l}\text { Los directivos están } \\
\text { conscientes de la nece- } \\
\text { sidad de GdC. }\end{array}$ & $\begin{array}{l}\text { Se documentan los } \\
\text { conocimientos in- } \\
\text { dispensables para la } \\
\text { realización de tareas } \\
\text { repetitivas. }\end{array}$ & $\begin{array}{l}\text { Se han iniciado } \\
\text { proyectos piloto } \\
\text { de GdC (no ne- } \\
\text { cesariamente por } \\
\text { iniciativa de los } \\
\text { directivos). }\end{array}$ \\
\hline 3 & Definido & $\begin{array}{l}\text { La organización ha } \\
\text { puesto en marcha una } \\
\text { infraestructura básica } \\
\text { que soporta la GdC. }\end{array}$ & $\begin{array}{l}\text { - Se proporciona forma- } \\
\text { ción básica sobre GdC. } \\
\text { - Se pone en marcha } \\
\text { una estrategia básica de } \\
\text { GdC. } \\
\text { - Se han definido roles } \\
\text { individuales de GC. } \\
\text { - Se han activado los } \\
\text { sistemas de incentivos. }\end{array}$ & $\begin{array}{l}\text { - Se han formali- } \\
\text { zado los procesos } \\
\text { para la gestión } \\
\text { de contenidos e } \\
\text { información. } \\
\text { - Los sistemas de } \\
\text { medición pueden } \\
\text { ser usados para } \\
\text { medir el incremento } \\
\text { de la productividad } \\
\text { por causa de GdC. }\end{array}$ & $\begin{array}{l}\text { - Se tiene insta- } \\
\text { lada una infraes- } \\
\text { tructura básica } \\
\text { de GdC (páginas } \\
\text { amarillas) } \\
\text { - Se han puesto } \\
\text { en marcha algunos } \\
\text { proyectos de GdC } \\
\text { en algunos niveles } \\
\text { de la pirámide } \\
\text { organizacional. }\end{array}$ \\
\hline 4 & $\begin{array}{l}\text { Gestionado, } \\
\text { establecido }\end{array}$ & $\begin{array}{l}\text { Las iniciativas de GdC } \\
\text { están plenamente } \\
\text { establecidas en la } \\
\text { organización. }\end{array}$ & $\begin{array}{l}\text { - Una estrategia común } \\
\text { y que apunta a la norma- } \\
\text { lización de la GdC. } \\
\text { - La GdC es incorporada } \\
\text { dentro de la estrategia } \\
\text { general de la organi- } \\
\text { zación. } \\
\text { - Formación avanzada } \\
\text { en GdC. } \\
\text { - Estándares organiza- } \\
\text { cionales. }\end{array}$ & $\begin{array}{l}\text { Medición cuantita- } \\
\text { tiva de los proce- } \\
\text { sos de GdC. }\end{array}$ & $\begin{array}{l}\text { - En toda la } \\
\text { organización los } \\
\text { sistemas de GdC } \\
\text { están funcionando } \\
\text { plenamente. } \\
\text { - El uso de los } \\
\text { sistemas de GdC } \\
\text { están en un nivel } \\
\text { razonable. } \\
\text { - Perfecta } \\
\text { integración de la } \\
\text { tecnología con la } \\
\text { arquitectura de } \\
\text { contenidos. }\end{array}$ \\
\hline 5 & $\begin{array}{l}\text { Optimizar, } \\
\text { compartir }\end{array}$ & $\begin{array}{l}\text { La GdC está plena- } \\
\text { mente integrada a la } \\
\text { organización y sometida } \\
\text { a procesos de mejora- } \\
\text { miento continuo. }\end{array}$ & $\begin{array}{l}\text { La cultura de compartir } \\
\text { está institucionalizada. }\end{array}$ & $\begin{array}{l}\text { - Los procesos de } \\
\text { GdC son revisados } \\
\text { permanentemente } \\
\text { y mejorados. } \\
\text { - Los actuales } \\
\text { procesos de GdC } \\
\text { pueden ser fácil- } \\
\text { mente adaptados } \\
\text { para satisfacer las } \\
\text { nuevas necesida- } \\
\text { des de negocio. } \\
\text { - Los procedimien- } \\
\text { tos de GdC forman } \\
\text { parte integral de la } \\
\text { organización. }\end{array}$ & $\begin{array}{l}\text { La infraestructura } \\
\text { actual de GdC es } \\
\text { mejorada conti- } \\
\text { nuamente. }\end{array}$ \\
\hline
\end{tabular}

Tabla 3. Modelo general de madurez de GC. Fuente: Pee et al. (2006). Traducción realizada por Durango et al. (2015). 
de retiro del personal a los 63 años de edad, flujo creciente de técnicos cursando niveles de especialización académica superior y cambios en las políticas de asignación de personal técnico a diferentes puestos) la organización identifica claramente la necesidad de optimizar la gestión del conocimiento, lo cual se constata en la información recabada durante la fase de relevamiento de los antecedentes del proyecto .

En el año 2014, el LATU tomó la decisión de implementar un SGdC incorporándolo como un objetivo de gestión. En el año 2007 un grupo ad hoc, integrado por técnicos de diferentes áreas, comenzó a trabajar en un diagnóstico sobre la temática. En el año 2009 se realizó una encuesta de $\mathrm{GdC}$, para la cual se convocó una muestra de 240 funcionarios efectivos de un total de 400, y logró una respuesta del $85 \%$, resultado que evidenciaba el interés por la temática.

La encuesta fue un primer acercamiento a la noción que los integrantes de la institución tenían sobre la gestión del conocimiento. Formó parte de la etapa de diagnóstico de la iniciativa institucional, que buscó obtener información objetiva y confiable sobre: el relacionamiento existente entre los distintos departamentos y los colaboradores; cómo se transfería el conocimiento proveniente de la capacitación recibida; las herramientas de archivo de información utilizadas; las herramientas y prácticas de gestión del conocimiento existentes en los diversos niveles de la estructura organizativa; los posibles beneficios que un proyecto institucional sobre la temática tendría para la organización, y las posibles barreras existentes.

A partir de los resultados obtenidos, el LATU identificó los beneficios relacionados con la realización de un proyecto de GdC en cuanto a aumentar las competencias, potenciar la innovación, estar mejor preparados para el cambio y mejorar las relaciones entre las diversas unidades de la estructura organizativa. Asimismo, se detectaron barreras relacionadas con la disponibilidad de tiempo del personal, los costos asociados a la asignación de recursos humanos en posibles funciones específicas y la resistencia a compartir conocimiento. Lo que ciertos autores denominan perturbación inducida por la tarea y perturbación inducida por el poder (Newell, 2005).

Considerando los antecedentes del LATU en relación a la temática (2007-2013), varias lecciones aprendidas fueron incorporadas a la toma de decisión de las futuras acciones que se emprendieron en el período 2014-2016. Entre ellas se destaca el activo involucramiento de la Gerencia General en el proyecto de diseño e implementación del sistema de $\mathrm{GdC}$, que participó en todas las actividades planificadas. Esta iniciativa fue fundamental para respaldar la importancia que la temática tenía y tiene para la dirección de la organización.

El proyecto de implementación del SGdC fue diseñado y ejecutado con el apoyo del Programa de Gestión del Conocimiento de la Universidad Católica del Uruguay. Se planificó para ser desarrollado en seis fases a lo largo de 32 semanas:

- Fase 0: Planificación del proyecto. Durante esta fase se acuerdan los diversos componentes del proyecto y la metodología a seguir, los roles y funciones asociadas, se intercambia con la consultoría sobre los colaboradores a participar en la fase siguiente y en las posteriores.

- Fase 1: Capacitación (ocho semanas). Este componente parte de unas primeras actividades de difusión, de sensibilización en la temática y de nivelación de conocimientos hasta concluir con la conformación de un grupo de trabajo específico.

- Fase 2: Consolidación del grupo de trabajo (cuatro semanas). Parte de este componente son: adoptar un marco de funcionamiento, establecer un acuerdo de trabajo, definir roles dentro del grupo, plantear la frecuencia de reunión, determinar las herramientas de evaluación de avances, adoptar un marco ético, teórico, técnico, estratégico y político, definir propósito y objetivos, plazos y productos entregables, y acordar la realización de una experiencia piloto exitosa y de impacto.

- Fase 3: Relevamiento de antecedentes (cuatro semanas). Se busca relevar la información en relación a la gestión del conocimiento en instituciones similares o comparables; la recuperación de conclusiones de los procedimientos, programas y estudios preliminares; el aporte de iniciativas propias anteriores (personales, grupales y organizacionales).

- Fase 4: Diagnóstico (cuatro semanas). El grupo de trabajo definido en la fase 2 realiza un diagnóstico en el que identifica el nivel de madurez, riesgos, condiciones favorables, limitaciones y viabilidad del diseño e implementación del SGdC.

- Fase 5: Diseño del Sistema de Gestión del Conocimiento (ocho semanas). A partir del diagnóstico realizado, el grupo diseña el SGdC a proponer, definiendo el alcance, los procesos relacionados, las prácticas, técnicas y herramientas a utilizar, y proponiendo acciones para superar las barreras y obstá- 
culos identificados en la fase 4 y en la comparación con las buenas prácticas internacionales en GdC.

- Fase 6: Piloto (cuatro semanas). Se acuerda realizar un piloto de implementación, que sea evaluado una vez finalizado.

Si bien esta última fase estaba prevista para el transcurso de 2015, no fue ejecutada ya que el LATU decidió realizar el diseño e implementación del SGdC para toda la organización en forma simultánea.

\section{Fase 1: capacitación}

Esta fase del proyecto fue diseñada y ejecutada en tres grandes módulos, en un diseño que se puede denominar en "embudo" en cuanto a su alcance; comenzó con todos los integrantes de la organización y culminó en la capacitación del grupo de trabajo específico.

En el primer módulo se realizaron talleres de sensibilización en la temática de gestión del conocimiento dirigido a todos los colaboradores efectivos (aproximadamente 350 personas). El segundo módulo estuvo dirigido a todas las gerencias, direcciones, coordinaciones y jefaturas, con participación de la Gerencia General (entre 60-70 personas). En el tercer módulo participó un grupo de aproximadamente 18 personas designadas por las gerencias que conformarían el grupo de trabajo para la implementación (Grupo $\mathrm{GdC}$ ), con representantes de todas las gerencias de la organización.

\section{Fase 2: consolidación del grupo de trabajo y adopción de un marco de funcionamiento}

Finalmente, a través de diferentes incorporaciones al núcleo de trabajo para la implementación, el grupo quedó conformado por 30 personas que representaban a todos los procesos de la organización.

Una de las primeras actividades del Grupo GdC fue generar un acuerdo de trabajo. En ese documento se refleja el compromiso de trabajo asumido por cada integrante del grupo en relación a su asistencia y cumplimiento de plazos con los objetivos del grupo; la frecuencia y horarios de las reuniones; la operativa de los grupos ad hoc; la toma de decisiones por consenso, y el establecimiento de un sistema de medición de avances, resultados parciales e impactos, que permitiera un seguimiento cercano del proyecto y mecanismos de difusión para informar del proyecto a la organización.

En esta fase se lograron los acuerdos básicos dentro del grupo para consensuar el significado que la ges- tión del conocimiento tiene para el LATU, la Misión de la GdC en el LATU y los objetivos organizacionales en relación al sistema.

A partir del contenido acordado en el acuerdo de trabajo surgió la necesidad de generar:

- Subgrupos con roles específicos durante toda la vida del proyecto,

- Grupos ad hoc en temáticas determinadas para elaborar propuestas que se consensuarían con el Grupo GdC.

Los subgrupos con roles específicos durante toda la vida del proyecto son:

- Grupo estado de situación y avance. Responsable del seguimiento del proyecto a lo largo de sus fases, cuentan con herramientas generadas en un grupo ad hoc constituido a tal fin.

- Equipo de comunicación. Con el objetivo de proponer y dar seguimiento a un plan de comunicación del proyecto y del sistema de gestión del conocimiento, establece instancias, actividades y medición de la efectividad de la comunicación tanto interna como externa.

Inicialmente, los grupos ad hoc creados para realizar propuestas concretas de avance al plenario en diferentes temáticas de interés fueron:

- Herramientas de evaluación de avance, cuyo objetivo era el seguimiento del proyecto (plazos y cumplimiento de hitos), la medición de resultados (diseño e implementación con cuestionarios, entrevistas, práctica), de efectos e impactos, lo cual se resume en la Figura 2.

- Marco ético. Dado que el LATU tiene un código de ética que abarca no solo a sus colaboradores sino también a sus proveedores, se consideró necesario revisar su contenido a partir de la generación del acuerdo de trabajo definido para el Grupo GdC. Del estudio realizado surgieron recomendaciones relacionadas con la propiedad intelectual del diseño del sistema de gestión del conocimiento así como de la confidencialidad de la información intercambiada durante el proyecto. 


\section{NVVELES DE MEDOCOON}

1. PLAZOS E HITOS

A) HERRAMIENTA: PROJECT SERVER

2. CALIDAD DEL RESULTADO

A) DEFINIDO PARA CADA MÓDULO

B) ACORDADOS ANTES DEL INICIO DEL MÓDULO

3. RESULTADO DEL EFECTO DEL PROYECTO

A) HERRAMIENTAS DESARROLLADAS

B) HERRAMIENTAS EMPLEADAS

C) EFECTIVIDAD DE LAS HERRAMIENTAS

4. IMPACTO

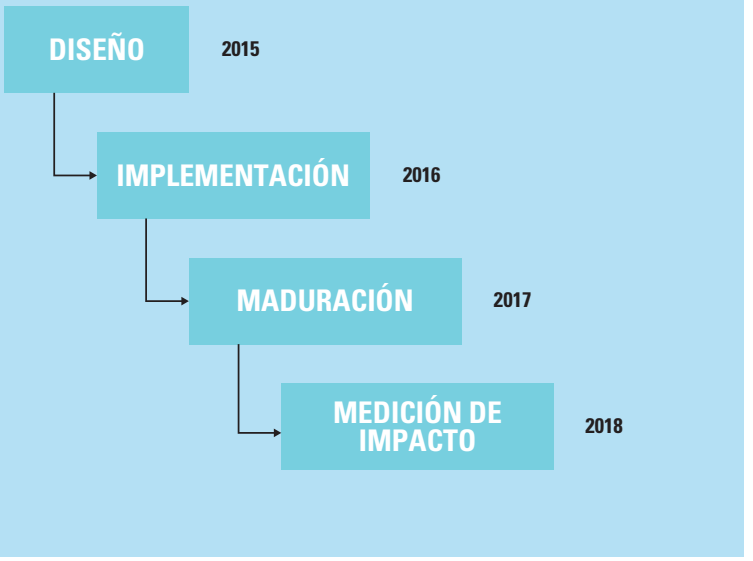

Figura 2. Niveles de medición de las fases del proyecto de implementación del sistema de gestión del conocimiento. Fuente: LATU, Grupo de gestión del conocimiento, año 2015.

\section{Fase 3: relevamiento de antecedentes}

El objetivo de esta fase fue capitalizar los esfuerzos que la organización ya hubiera realizado en relación a la gestión del conocimiento, pero sin asociarla conscientemente a ese concepto, buscando líneas de acción a partir de las sugerencias o propuestas que en su momento se hubieran hecho.

El Grupo GdC realizó un relevamiento de todos los antecedentes significativos para el proyecto, e identificó 10 áreas de interés. Se definieron cuatro grupos ad hoc para relevar la información. Estos grupos detectaron fuentes, resultados y acciones preexistentes en el LATU que aportaban a la GdC ya que contribuían a desarrollar las condiciones para que las personas produjeran, aplicaran y/o transfirieran un conocimiento válido para la organización.

El resultado del análisis se resumió en una matriz de elementos que se contemplarían e integrarían al sistema de gestión del conocimiento.

\section{Fase 4: diagnóstico}

Las conclusiones del relevamiento de antecedentes alimentaron la siguiente fase de diagnóstico específico sobre la GdC en el LATU, que incluyó la aplicación de un Modelo de Madurez, adaptado para este proyecto, desarrollado en un equipo ad hoc a partir de la revisión bibliográfica. De los modelos analizados, se adoptó para la evaluación de la madurez de la organización uno indicado como proveniente de "fuentes varias". Sobre este esquema se trabajaron algunos ítems con aportes provenientes de otros modelos, con el fin de que la herramienta ganara en sensibilidad y claridad en las descripciones.

El modelo de madurez adaptado dispone de seis dimensiones (alineación estratégica, liderazgo y gobernanza, organización y procesos, contenido y contexto, cultura y personas, y tecnología), distribuidas en cinco niveles evolutivos (gestión ignorada, gestión incipiente, gestión parcial, conocimiento gestionado y conocimiento optimizado), y totaliza 100 escenarios posibles.

El modelo fue aplicado a todos los procesos del LATU, y se obtuvo como resultado una matriz de doble entrada.

La responsabilidad por la aplicación del modelo fue asignada a los responsables de los procesos (niveles gerenciales), quienes decidieron individualmente la metodología utilizada para su aplicación (individual, en consulta y/o en equipo con colaboradores más cercanos, por ejemplo). Asimismo, fue acordado que cada proceso indicara su percepción en relación al desarrollo de la GdC, del propio proceso y de la organización en su conjunto. Es decir, cada proceso realizó un diagnóstico de sí mismo y otro de la organización. 
En el Grupo GdC se describen y analizan los promedios, dispersión y brechas resultantes de la aplicación del modelo de madurez utilizado. A su vez, aquellos procesos que se percibieron como ubicados en fases posteriores de desarrollo pudieron explicar a los otros procesos cómo creían haberlo logrado. Y los procesos que se autodefinieron o reconocieron estar en estadios anteriores de la GdC pudieron preguntar, o requerir de los otros procesos, qué hacer para desarrollarse.

Los resultados fueron consolidados por un equipo ad hoc que sintetizó las principales conclusiones a las que arribaron y que fueron presentadas al equipo plenario para su validación. Las conclusiones se consignaron de acuerdo a la fuente de la que provenían, ya sea de la aplicación del modelo de madurez adoptado o del relevamiento de antecedentes.

Las conclusiones de la fase de diagnóstico fueron utilizadas para nutrir al Sistema de GdC que se diseñó durante la siguiente fase del proyecto.

\section{Fase 5: diseño del sistema de gestión del conocimiento}

Con base en las conclusiones resultantes de la fase de diagnóstico, el Grupo GdC acordó diseñar un SGdC con ciertas características prioritarias incluidas en su propósito, alcance, estructura, responsabilidades y prácticas a desarrollar. Durante la fase 2 del proyecto se consensuaron: la definición de GdC, su misión y los objetivos principales relacionados, y en esta fase solo fue necesario relacionar el SGdC con esas definiciones.

El alcance del SGdC fue definido para todos los procesos identificados en el mapa de procesos del LATU, e incluye todos los subprocesos característicos de la gestión del conocimiento:

- Generación: creación, desarrollo, investigación, innovación, acceso, adopción, adaptación, aprendizaje por experiencia, capacitación (entrenamiento, especialización, actualización, recapacitación, formación), captura exterior (acceso, reclutamiento).

- Intercambio: socialización, exteriorización, interiorización, combinación, transferencia.

- Identificación: selección, ubicación, exploración (vigilancia, benchmarking).

- Conservación: protección, confidencialidad, propiedad, derechos.
Como fue mencionado, el SGdC es responsabilidad del proceso de Dirección del LATU, el cual puede asignar contrapartes para cada proceso de la organización. A su vez, cada uno de esos procesos, en coordinación con el SGdC, es responsable por la ejecución y seguimiento de las prácticas que se implementan en su ámbito. Aquellas prácticas, técnicas o herramientas de GdC que sean de uso general o de uso exclusivo de un proceso pueden contar para su implementación y gestión con un equipo ad hoc integrado con colaboradores de distintos procesos.

Las prácticas de conocimiento a desarrollarse (continuarse, retomarse o crearse), definidas a partir de los resultados del diagnóstico realizado en la fase anterior, fueron documentadas en una matriz y se establecieron plazos (inmediato, corto y mediano) y procesos necesariamente involucrados, así como su responsable. En total se identificaron 33 medidas.

Para la implementación de las prácticas de conocimiento con plazos definidos como inmediato y corto (19 prácticas) se conformaron seis subgrupos de trabajo con integrantes pertenecientes a diferentes procesos, previamente propuestos por la coordinación del proyecto, si bien se indicó que los grupos eran abiertos y de participación voluntaria. La coordinación del proyecto definió un coordinador por cada subgrupo. Un séptimo subgrupo, responsable por el diseño del proceso de dirección del SGdC, estuvo integrado por todos los gerentes responsables por los procesos incluidos en el mapa de procesos del LATU.

Los siete subgrupos fueron creados con el objetivo de diseñar las prácticas de conocimiento que le fueran asignadas. Los subgrupos tienen la responsabilidad de realizar los diseños correspondientes, que luego son acordados a nivel de todos los integrantes del Grupo de GdC. Los documentos resultantes son aprobados por los responsables de los procesos involucrados en las acciones relativas a la implementación.

\section{Algunas lecciones aprendidas}

A partir de los resultados obtenidos por la introducción del sistema de $\mathrm{GdC}$ han sido identificadas conclusiones asociadas al proceso de diseño e implementación que se resumen en los próximos seis apartados: necesidad de participación, adquisición de un lenguaje técnico común, construcción de un sistema propio, capitalización de antecedentes, búsqueda de consensos y respeto de la cultura organizativa. 


\section{Necesidad de participación}

Hoy en día todos los autores coinciden en afirmar que una $\mathrm{GdC}$ exitosa se logra no solo satisfaciendo las necesidades de la organización, sino también las necesidades individuales de sus integrantes y de las comunidades de práctica que la integran. Para conocer las necesidades individuales de sus integrantes es necesario que las personas participen, que tengan interés en la temática y que aporten su experiencia, tomando conocimiento de qué es lo que los otros saben, y haciendo posible así superar las dificultades relacionadas con una de las características principales del conocimiento: la dispersión.

En el transcurso del proyecto, el interés fue evidenciado a partir del excelente nivel de participación de todos los colaboradores convocados, quienes compartieron sus conocimientos para el logro de los objetivos consensuados, y que fueron proactivos en el trabajo de los equipos. La concurrencia de los integrantes del grupo de trabajo a las reuniones superaba el $80 \%$ de asistencia y la de los grupos ad hoc, el $90 \%$.

Por la propia esencia del conocimiento, que reside en las personas, quienes a su vez lo comparten ya sea en forma explícita o tácita, todos los colaboradores deben tener la vivencia del proceso de diseño e implementación del SGdC, transitar por él y participar activamente en su definición, directa o indirectamente.

Si las personas no participan, seguramente tiendan a sentir mayor rechazo frente a los cambios que las involucren, y presenten como consecuencia una falta de compromiso y de motivación por aquello que les sea "impuesto". La participación es parte esencial de la gestión del cambio, en tanto que a mayor participación habrá menor resistencia.

El conocimiento disperso debe reunirse para que se establezca una comprensión conjunta del sistema y, por ende, una adecuada reflexión que permita la generación de nuevas ideas acerca de cómo deben hacerse las cosas.

La implementación de un SGdC tiene características comunes a todos los sistemas de gestión de una organización, ya que requiere el compromiso de la alta dirección para aumentar las probabilidades de éxito del proyecto. El intercambio de ideas y percepciones entre la dirección con colaboradores de diferentes niveles jerárquicos, no solo evidencia la importancia que para la institución tiene la temática, sino que permite que ciertos temores que coexisten con la implementación de estos sistemas (como la percepción de pérdida de poder) sean minimizados.
Es imprescindible relacionar la $\mathrm{GdC}$ con las estrategias de la organización, promoviendo que los colaboradores identifiquen frecuentemente las ventajas de compartir conocimiento.

\section{Adquisición de un lenguaje técnico común}

Las instancias de capacitación, entre otras, son eventos útiles para nivelar el conocimiento de las personas sobre las diferentes temáticas y permiten la adquisición de un lenguaje técnico común.

Este lenguaje es indispensable para consensuar el significado que la gestión del conocimiento tiene para la organización, la propia misión de la GdC en la institución y los objetivos organizacionales en relación al sistema.

El conocimiento es ambiguo, por lo que diferentes personas o grupos pueden interpretar un concepto o práctica de modo distinto, según sus propios paradigmas, que a su vez restringen la validez del conocimiento. Se deben considerar las barreras sintácticas y semánticas para compartir el conocimiento y desarrollar interpretaciones comunes para superarlas (Nonaka, 1994). Esto permitirá que se reflexione sobre las diferentes interpretaciones y que esta reflexión presente nuevos conceptos para alcanzar el consenso acerca de cómo hacer las cosas de un modo diferente.

\section{Construcción de un sistema propio}

Si la GdC de una organización se desarrolla teniendo como pilares básicos la participación de todo el personal compartiendo un lenguaje técnico común, es factible la construcción de un sistema propio, adecuado a las necesidades, fortalezas y debilidades de la propia organización, con altas probabilidades de éxito en su desempeño.

En la implementación del SGdC es importante resaltar la práctica de no trasplantar sistemas diseñados para otras organizaciones, aunque se los considere altamente exitosos. La propia esencia del conocimiento (el hecho de que reside en las personas) implica la necesidad de que la organización construya un sistema propio basado en sus características particulares.

El diseño e implementación de SGdC debe ser gestionado en el marco de un proyecto (o cartera de proyectos) de la organización, así como también debe 
incluir la planificación, la asignación de responsabilidades y de recursos para su ejecución, el seguimiento y control de avance para identificar las acciones correctivas requeridas, y la replanificación de fases, etapas y actividades.

\section{Capitalización de antecedentes}

Tradicionalmente, las áreas del LATU se gestionaron con un cierto grado de "duplicación de los especialistas". Todo profesional universitario que desempeñara grado de jefaturas (grado de mayor especialización técnica) tenía un alterno (profesional universitario) con similar nivel de especialización o en formación. Ello permitía que, en caso de ausencia del titular, su alterno pudiera brindar una respuesta técnica de similar nivel de especialización.

Es interesante comparar esta práctica con el principio de redundancia estudiado por Nonaka y Takeuchi en las compañías japonesas (Nonaka y Takeuchi, 1995), por el cual las organizaciones conscientemente duplican información, conocimientos, actividades empresariales o responsabilidades de gestión, lo que facilita la transferencia de conocimiento tácito a través del fortalecimiento del diálogo y la comunicación e interacción entre los integrantes de la empresa. Indudablemente, la decisión organizativa de contar con jefatura y alterno estimulaba el diálogo y facilitaba la transferencia del conocimiento tácito entre uno y otro.

Actualmente, el principio de redundancia es escaso dentro de la institución. La estrategia de optimización de recursos, incluso los relacionados con su capital intelectual, exige a los cargos de gestión desafíos importantes para obtener mejores resultados en relación al aprendizaje organizacional, la retención de talentos y la innovación.

El relevamiento de antecedentes permite capitalizar los esfuerzos que la organización haya realizado pero sin asociarlos conscientemente a la gestión del conocimiento, buscando líneas de acción a partir de las sugerencias o propuestas realizadas. Es de vital importancia desarrollar un SGdC propio, nacido de las experiencias y de las lecciones aprendidas de los integrantes de la organización.

Las áreas de interés para realizar el relevamiento de antecedentes deben ser significativas e identificarse en forma específica para cada organización. Es esperable que la organización genere grupos ad hoc en el equipo asignado al diseño del sistema, con el fin de recabar en paralelo los diversos antecedentes y optimizar plazos. Una vez obtenida la información, el tiempo que se planifique en el proyecto para difundir los trabajos e iniciativas relevadas entre todos sus integrantes debe ser suficiente para lograr una adecuada profundidad de análisis, que enriquezca el intercambio en el equipo.

Durante la socialización del conocimiento resultante de la aplicación del modelo de madurez, los procesos que se percibieron ubicados en niveles altos de desarrollo compartieron con los demás cómo creían haberlo logrado. En tanto los procesos que se autoidentificaron en estadios anteriores pudieron preguntar o requerir qué hacer para mejorar su desarrollo.

\section{Búsqueda de consensos}

La decisión por consenso es un proceso de decisión que busca no solamente el acuerdo de la mayoría de los participantes, sino que también persigue el objetivo de resolver o atenuar las objeciones de la minoría para alcanzar la decisión más satisfactoria (Enebral, 1998).

Los sistemas participativos, el trabajo en equipo, el liderazgo, el empoderamiento y, en general, todas las nuevas tendencias del management, parecen apuntar al consenso como medio de asegurar la mayor eficiencia en la aplicación de las decisiones adoptadas. Es una especie de management by consensus.

En el diseño e implementación de un SGdC, la búsqueda de consensos por parte de la mayor cantidad posible del personal de la organización debería ser un objetivo del propio proyecto de implantación. La naturaleza del conocimiento, que reside en las personas, condiciona a la organización a la búsqueda de consensos para viabilizar en el transcurso del tiempo el funcionamiento de los elementos críticos del sistema de GdC.

En la búsqueda del mayor consenso posible se resalta la participación de los delegados gremiales. Dos integrantes designados por el propio gremio son miembros activos del Grupo GdC, y participaron en los grupos ad hoc generados para el abordaje de diversas iniciativas. Sus aportes e inquietudes son tenidos en cuenta en el diseño del SGdC, posibilitando la mayor generación de confianza sobre su contenido. 


\section{Respeto por la cultura organizativa Conclusiones}

Newell (2005) hace referencia a la perturbación inducida por la cultura, que puede llegar a impedir o suboptimizar la creación y transferencia de conocimiento como consecuencia de la influencia ejercida por los valores o creencias propios de la cultura de la organización.

Es importante que el diseño e implementación de un SGdC no solo promueva una cultura de compartir el conocimiento, sino que identifique y respete los demás elementos de la cultura organizativa que coexisten con la cultura del conocimiento.

Si, por ejemplo, la organización tiene una cultura jerárquica (Cameron y Quinn, 1999), se debe tener en cuenta que sus valores más importantes son la eficiencia, el cumplimiento de las normas y la formalización de los procesos. Por lo tanto, el SGdC más adecuado será aquel que haya sido diseñado contemplando estas características pero, a su vez, introduciendo los elementos característicos de una cultura de compartir conocimiento. En esta cultura, el trabajo en equipo, el colaborar y compartir y los reconocimientos por hacerlo seguramente se encuentren formalmente establecidos en la documentación del sistema asociada a los procesos de la organización.

En cambio, frente a una cultura adhocrática en la que la iniciativa, la creatividad y la asunción de riesgos son valores importantes, el trabajo en equipo, el colaborar y compartir y los reconocimientos por hacerlo seguramente tengan una flexibilidad casi desconocida y no compartida por una organización de cultura jerárquica.

Asimismo, es importante tener en cuenta que una organización no cuenta con una única cultura. Usualmente coexisten subculturas que se diferencian de la cultura principal de la organización y de las otras subculturas existentes (Cameron y Quinn, 1999).

Por ello es importante la necesidad de tomar consciencia de las diferentes culturas organizativas dentro de una misma organización, para proponer o diseñar las herramientas de GdC más adecuadas a sus características particulares. Lo importante es no perder el propósito buscado: desarrollar una cultura que incentive e impulse la gestión del conocimiento.
El artículo analiza el proceso de diseño e implementación de la gestión del conocimiento en el LATU. La implementación del sistema recién ha comenzado y ha tenido que sortear varias barreras culturales, tecnológicas, organizativas, de ausencia de conocimientos específicos, entre otras, que nos revelan que el camino hacia la cultura de la Gestión del Conocimiento no es sencillo.

EI LATU comprendió la importancia de gestionar el conocimiento de la organización, para seguir siendo un referente en innovación y transferencia tecnológica y aumentar su competitividad.

Para ello se entiende imprescindible relacionar la GdC con las estrategias de la organización, promoviendo que los colaboradores identifiquen frecuentemente las ventajas de compartir conocimiento y participen activamente con sus aportes al SGdC.

Las palabras del Gerente General del LATU resumen la esencia del SGdC implementado: "Para el LATU el conocimiento constituye uno de los activos fundamentales. Nuestros colaboradores son los articuladores de los servicios que ofrece la institución, así como representantes de su misión de innovación y transferencia de tecnologías. Para una institución como la nuestra, cuyos productos y servicios se basan en los conocimientos de los colaboradores, la gestión del capital humano requiere de un ambiente laboral que favorezca el intercambio de conocimiento, la convivencia humana y la creatividad; de esta forma, se logra el desarrollo y crecimiento no solo de la institución, sino especialmente de las empresas uruguayas. Alineados a nuestra misión institucional, hemos incorporado un sistema de gestión del conocimiento integrado a los otros sistemas de gestión (calidad, salud y seguridad, entre otros). Está diseñado con el propósito de gestionar el conocimiento organizativo como un activo estratégico. Es así que la gestión del conocimiento para el LATU es el proceso sistémico que permite crear, incorporar y gestionar el conocimiento necesario, reflejándolo en servicios y soluciones de valor" (Silveira, 2017). 


\section{Reconocimientos}

Se desea agradecer al equipo de Gestión del Conocimiento del LATU por su contribución en el diseño y desarrollo del Sistema de Gestión de Conocimiento del LATU, a la coordinadora del Proyecto MBA, Ing. Mariela De Giuda, y al PhD. Fernando Zeballos por sus valiosos aportes al proyecto de Gestión del Conocimiento.

\section{Referencias}

Asociación Española de Normalización y Certificación, 2008. UNE 412001: Guía práctica de gestión del conocimiento. Madrid: AENOR.

Bloom, H., 2000. The global brain. New York: John Wiley and Sons.

Boisot, M., 1998. Knowledge assets. Oxford: Oxford University Press.

Bukowitz, W. y Williams, R., 2000. The knowledge management fieldbook. London: Prentice Hall.

Cameron, K. y Quinn, R., 1999. Diagnosing and changing organizational culture. New York: Wiley John and Sons.

Dalkir, K., 2005. Knowledge management in theory and practice. Montreal: McGill University.

Di Candia, C., Pippolo, D. y Rainusso, V., 2011. ¿Por qué conocer la cultura de una organización? En: INNOTEC Gestión, 3, pp.54-63.

Durango, C., Quintero, M. y Ruíz, C., 2015. Metodología para evaluar la madurez de la gestión del conocimiento en algunas grandes empresas colombianas [En línea]. En: Tecnura, 19(43): pp.20-36. [Consulta: 18 de diciembre de 2017]. Disponible en: http://dx.doi. org/10.14483/udistrital.jour.tecnura.2015.1.a01

Enebral Fernández, José, 1998. Decisiones por consenso. En: Revista Capital Humano, Año XI (112), pp.64-66.

Gruber, H. 2000. Does organizational cultura affect the sharing of knowledge? [En línea]. Otawa: Carleton University. (Tesis de Maestría) [Consulta: 18 de diciembre de 2017]. Disponible en: https://curve. carleton.ca/806face2-3cf7-4404-ba4c-713e1798be24
Harmon, Paul, 2009. Process maturity models [En línea]. En: BPTrends, 2(5). [Consulta: 12 de junio de 2016]. Disponible en: http://www.bptrends.com/ publicationfiles/spotlight_051909.pdf

Lee Blanco, Carlos Alfonso, 2012. El capital intelectual y las redes de conocimiento. En: INNOTEC Gestión, 4, pp.22-29.

Mazur, G., 1993. QFD for service industries: from voice of customer to task deployment. Michigan: Japan Business Consultants.

Meyer, M. y Zack, M., 1996. The design and implementation of information products. En: Sloan Management Review, 37(3), pp.43-59.

Mc.Elroy, M., 2003. The knew knowledge management: complexity, learning and sustainable innovation. Boston: Butterworth-Heinemann.

Newell, S., 2005. Knowledge transfer and learning: problems of knowledge transfer associated with trying to short-circuit the learning cycle. En: Journal of Information Systems and Technology Management, 2(3), pp.275-290.

Nonaka, I., 1994. A dynamic theory of organizational knowledge creation. En: Organization Science, 5(1), pp.14-37.

Nonaka, I. y Takeuchi, H., 1995. The knowledge-creating company: how Japanese companies create the dynamics of innovation. New York: Oxford University Press.

Organisation for Economic Co-operation and Development, OECD, 2000. Knowledge managemengt in the learning society. Paris: OECD.

Organisation for Economic Co-operation and Development, OECD, 2004. Measuring knowledge management in the business sector. Paris: OECD.

Pee, L., Teah, H. y Kankanhalli, A., 2006. Development of a general knowledge management maturity model. Seúl: Korean Knowledge Management Society Conference. pp. 17-18. 
Ruggles, R., 1977. Knowledge tools: using technology to manage knowledge better. Boston: ButterworthHeinemann.

Senge, Peter, 1990. La quinta disciplina. El arte y la práctica de la organización abierta al aprendizaje. Madrid: Granica.

Silveira, Jorge, 2017. ¿Por qué desarrollar un sistema de gestión del conocimiento en el LATU? [En línea]. En: El Observador, 10 de marzo de 2017. [Consulta: 18 de diciembre de 2017]. Disponible en: http://deres.org.uy/ wp-content/uploads/deres-1-3.pdf

Snowden, D., 2002. Complex acts of knowing: paradox and descriptive self-awareness. En: Journal of Knowledge Management, 6(2), pp.100-111.

Sveiby, K. y Simons, R., 2002. Collaborative climate and effectiveness of knowledge work-an empirical study. En: Journal of Knowledge Management, 6(5), pp.420-433.

Uruguay. Ley 18.640, de 26 de enero de 2010. Diario Oficial, 26 de enero de 2010, No. 27.905, p. 225A

Wiig, K., 1993. Knowledge management foundations. Arlington: Schema Press. 